\title{
Le tourisme, matière à penser de la science géographique
}

Olivier Lazzarotti

\section{(2) OpenEdition}

12 Journals

Édition électronique

URL : http://journals.openedition.org/tourisme/314

DOI : 10.4000/tourisme.314

ISSN : 2492-7503

Éditeur

Éditions touristiques européennes

\section{Édition imprimée}

Date de publication : 1 juin 2010

Pagination : 7-16

ISSN : 2109-5671

\section{Référence électronique}

Olivier Lazzarotti, « Le tourisme, matière à penser de la science géographique », Mondes du Tourisme

[En ligne], 1 | 2010, mis en ligne le 30 septembre 2015, consulté le 19 avril 2019. URL : http:// journals.openedition.org/tourisme/314; DOI : 10.4000/tourisme.314

\section{(c) $($ () $\ominus$}

Mondes du tourisme est mis à disposition selon les termes de la licence Creative Commons Attribution - Pas d'Utilisation Commerciale - Pas de Modification 4.0 International. 


\title{
Le tourisme, matière à penser de la science géographique
}

\author{
OLIVIER LAZZAROTTI \\ Professeur de géographie • Directeur de l'équipe d'accueil \\ "Habiter : processus identitaires, processus sociaux" • Université \\ de Picardie Jules Verne [olivier.lazzarotti@u-picardie.fr]
}

Résumé. Cet article analyse tout d'abord l'émergence, la constitution et l'évolution des études sur le tourisme, essentiellement du point de vue de la géographie française, le cas échéant dans ses relations avec les autres sciences engagées. Parti de là, il en présente les dernières tendances, représentées par le mouvement d'une approche géographique du tourisme et quelques-unes de ses perspectives heuristiques : critique des mots par les lieux, approche par les pratiques, importance de l'historicité des lieux. Inspirant le concept d'habiter, ces réflexions peuvent, en retour, en susciter d'autres, non exclusives, existentielles, politiques, économiques et mémorielles. Ainsi abordé, le tourisme offre à la science géographique le champ ouvert de sa manifestation, à l'articulation des données phénoménales, matières et pratiques, qui en constituent les donnés de base et celles de leur interprétation sociale.
Abstract. This article analyses the emergence, the constitution and evolution of the research on tourism from the point of view of French geography and its relationships with other disciplines. It presents the latest trends, especially the movement towards a "geographical approach of tourism", and the essential elements of its heuristical perspectives: criticism of words through places, practicecentred approach, importance of the historicity of places. Inspiring the concept of "dwelling", those reflections are able to trigger others, such as existential, political, economical and memorial issues. Thus, tourism offers to the geographical science an open range of its manifestations, linking various phenomena, materiality and practices and their social interpretations. 
$\mathbf{L}$ a relation entre le tourisme pratique à la forte dimension spatiale - et la géographie française est à la fois ancienne et privilégiée. Et le fait que les géographes eux-mêmes, à l'occasion en tant que touristes, en fassent personnellement l'expérience renforce très probablement ce lien : parler de tourisme, n'est-ce pas aussi faire de la géographie, et réciproquement ? Dès lors, on comprend qu'ils aient pu aborder très tôt cette question, même si leurs premiers textes ne relèvent pas de l'analyse purement scientifique, pas plus qu'ils ne ressemblent aux guides habituels en la matière, les Guides Bleus à l'époque. On pense, entre autres, au texte de Maurice Le Lannou mettant en scène, suivant les principes de sa démarche géographique, le pays de son enfance. On le découvre alors en véritable instituteur du territoire breton (Le Lannou, 1938).

La présente réflexion vise à aborder, rapidement, l'histoire des relations réciproques entre une expérience de géographie spontanée, le tourisme, et sa mise en mots et en notions par les tenants de la science géographique (Lazzarotti, 2002), mais aussi à présenter l'apport de ce phénomène aux dynamiques scientifiques pour soulever, finalement, quelques perspectives contemporaines, voire à venir. Le choix est contestable mais, dans les limites de ce bref travail, les études porteront essentiellement sur la géographie française.

\section{TOuRISME, QUELLE DÉFINITION ?}

Le tourisme fait son entrée en science sous le signe paradoxal d'une accumulation de connaissances autour d'un objet dont la définition ne cesse de poser problème.

\section{Le temps des thèses d'État}

L'entrée du tourisme dans le champ géographique académique date des années 1950, très généralement via les études urbaines, alors peu dominantes, notons-le (Chabot, 1957), ou via celles portant sur la circulation (Capot-Rey, 1947). Prenant en compte le temps des recherches, il émerge fortement dans les années décennies. C'est le temps des premières accumulations, dans des cadres épistémologiques qui restent ceux de la géographie française, c'est-àdire qu'il est abordé, certes, mais selon les entrées classiques de la géographie institutionnelle.

Elles sont celles de la géographie régionale, le plus souvent à fondement physique, comme les côtes de France (Burnet, 1957), la Costa Brava (Barbaza, 1966), les Landes de Gascogne (CassouMounat, 1977), la Normandie (Clary, 1977), l'Ouest français (Bonneau, 1978), ou les Alpes autrichiennes (Herbin, 1980), etc. Cela dit, le tourisme fait aussi l'objet d'études dans des régions où il n'était pas évident, selon les critères courants et partagés du moment, de le trouver. Tel est le cas de l'axe rhénan (Wackermann, 1973), de la région du Nord-Pas-de-Calais (Dewailly, 1985) ou dans celles des Pays de la Loire abordée, à sa manière, dans le travail de Mirloup (198I). Nous leur sommes redevables à la fois de cette ouverture et de la réflexion engagée sur les relations entre tourisme et loisirs (Lazzarotti, 1995).

Le déplacement est encore plus clair dans le cas des pays alors dits du tiers-monde, ouvrant à l'occasion une première collection dédiée aux tourismes aux éditions l'Harmattan (Cazes, 1989). Cette thématique aura de belles suites : les travaux de Berriane (1992) et
d'Hillali $(2003,2007)$ au Maroc ; ceux de Miossec (1996) en Tunisie, etc. D'autres études, encore, optent pour des approches plus spécifiquement thématiques. Ce sont, entre autres, celles de Cribier (1969), qui privilégie l'entrée dans le champ par les mobilités, ou de Knafou (1978), qui adopte celle d'un lieu spécifiquement touristique, les stations intégrées de sports d'hiver.

Quant à la dimension économique, elle était l'objet d'une attention particulière (Ginier, 1969). Elle sera reprise sous forme d'un ouvrage maintes fois réédité depuis 1992, celui de Py (2007).

Cela dit, durant cette période, le tourisme demeure malgré tout globalement un sujet à la fois dominé et hétéronome. Je pourrais, plus personnellement, en témoigner encore. Lors d'un entretien avec Étienne Dalmasso concernant mon projet doctoral - c'était au tout début des années 1990 -, celui-ci me dit avoir eu l'idée de faire sa thèse d'État sur le tourisme en Côte d'Azur, idée dont le détourna alors Pierre George, au profit de Milan et de son économie. Son étude sur Nice restera ainsi sans lendemain (Dalmasso, 1963)...

\section{Le temps des synthèses}

Le sujet aurait-il mûri ? Au cours des années 1980, vint le temps des synthèses. On assista alors à l'autonomisation du champ, en même temps qu'à sa reconnaissance institutionnelle, celle de la "géographie du tourisme", dont atteste la sortie de plusieurs manuels autant destinés à l'enseignement qu'à la recherche.

Chacun choisit son entrée et la développe. Au premier titre paru, fondé sur une approche morphologique (Lozato, 1985), succède un 
autre, d'orientation plus méthodologique (Cazes, 1992), puis un autre, enfin, cultivant la logique systémique (Clary, 1993). Dernier de cette série, le manuel de Dewailly et Flament (1993) produit une lecture dynamique du phénomène, avant une seconde édition (2000) qui, finalement, met en relief les impasses qui sont désormais celles de cette démarche, en particulier en matière de définition du tourisme. Expliquant qu'il est bien difficile, voire impossible, de définir le tourisme, les auteurs se retranchent finalement derrière la définition la plus froidement comptable, celle de l'OMT (pour sa version internationale) ou d'Odit France. Cela dit, la voie est tracée et tous ces travaux auront bien installé le tourisme dans le champ académique. Le marché ouvert, d'autres manuels suivront alors. Certains tentent un éclairage spécifique en croisant le tourisme avec d'autres thèmes, l'aménagement par exemple à partir du tourisme (Dewailly, 1990), voire le tourisme à partir de l'aménagement (Merlin, 200I). L'environnement y trouve son compte (Michaud, 1983), puis le climat (Besancenot, 1990) puis la montagne (Debarbieux, 1995), etc. D'autres mettent sous une forme pédagogique les résultats de recherches plus scientifiques (Duhamel et Sacareau, 1998), à côté d'une production qui fait entrer le genre dans son classicisme (BaronYellès, 1999). Dans un tel contexte, l'ouvrage de Pearce (1993), publié originellement en 1987, et dans sa traduction française en 1993, trouve ainsi "naturellement" toute sa place.

La géographie du tourisme se trouve ainsi structurée, et le thème du tourisme est alors croisé avec quelques-unes des grandes notions de la discipline. Pourtant, le champ reste quand même un peu bancal. C'est que la définition même du tourisme est rarement abordée autrement qu'à travers sa comptabilité. Du coup, on assiste à une déclinaison catégorielle du phénomène, qui le démultiplie et, à sa manière, l'embrouille en négligeant ce qui, au-delà de toutes ses manifestations, pourrait aussi le constituer. Le tourisme est vert (Béteille, 1996), urbain (Cazes et Potier, 1998), local (Amirou et Bachimon, 2000), bleu, sportif, religieux, etc., bref tout peut être tourisme. Un second travers tient à des appréciations, parfois implicites, mais qui participent à conditionner le regard que l'on peut porter sur lui. Le tourisme devient "sexuel", suggérant que le fait de faire du tourisme transforme, ipso facto, un "bon père de famille" en pédophile affamé.

\section{L'émergence d'une approche géographique du tourisme}

La formulation de ces critiques accompagne l'émergence d'une autre vision, et de la géographie et du tourisme, qui négocie alors le passage de la géographie du tourisme à l'approche géographique du tourisme.

Celui-ci est opéré par Knafou et l'équipe MIT. Dès lors, il s'agit de considérer la dimension géographique du phénomène, pour la cerner et la rapporter aux autres, historique, sociologique, etc. Les thèses de Deprest (1997), Duhamel (1997) et Stock (200 I) en sont directement issues. Des publications collectives et à géométrie variable suivront (Équipe MIT, 2002, 2005). Elles trouveront, dans un manuel universitaire, une formulation pédagogique (Stock, 2003). Tous ces textes profitent alors du dynamisme des éditions Belin qui ouvrent, avec la collection
Mappemonde, inaugurée par le livre de Florence Deprest (1997), un autre lieu de publication pour ces travaux. Fin 2008 arriveront, chez le même éditeur, les premiers volumes d'une collection de manuels universitaires dédiés au tourisme (Violier, 2008).

Il résulte de tout cela non seulement un important effort de définition du tourisme, mais encore une nouvelle manière de le définir, faisant primer la densité sur l'accumulation d'épithètes : quel peut être le point commun entre ces différentes manières de faire du tourisme ? Second point, la définition se veut pratique : ce ne sont pas les espaces, même touristiquement aménagés, qui, à eux seuls, font le tourisme. Ce sont les touristes qui, par leur présence, définissent un lieu comme touristique. Il faudra donc s'attacher à définir un touriste, en particulier à partir de son type de mobilité, car ce qui marque le tourisme est le déplacement, le changement de lieu. Le rapport au temps doit encore être pris en compte : le touriste se déplace dans un temps précis qui est celui du hors-quotidien. Cela permet de distinguer le tourisme des loisirs, pris quant à eux dans le temps du quotidien. Enfin, le touriste ne se déplace pas en vain. Il le fait en fonction d'une intentionnalité, celle de se recréer, de découvrir ou de jouer, tout cela permettant de distinguer le tourisme du voyage d'affaires. Enfin, croisant espace et temps, cette définition permet une lecture dynamique, autrement dit le passage d'une pratique de loisirs, comme en "amont" du tourisme, aux formes de nouvelles pratiques résidentielles, comme à son "aval", quand le déplacement est important, mais régulièrement opéré. Cette définition pratique du tou- 
risme, autrement dit fondée sur les pratiques des touristes, confirme ce que l'usage courant du vocabulaire réitère à tout bout de champ : bel et bien, on fait du tourisme.

La participation au Dictionnaire de la géographie et de l'espace des sociétés marque une nouvelle étape, autant dans la dynamique du groupe que dans la réflexion qui en émerge. Dans une acception plus globale et bouclée, mais qui, peut-être aussi, finalise une démarche en l'achevant, le tourisme y est considéré (2003, p. 931) comme un "système d'acteurs, de pratiques et d'espaces qui participent à la 'recréation' des individus par le déplacement et l'habiter temporaire hors des lieux $d u$ quotidien". Il s'en dégage le lien important et, à l'époque, guère étudié, entre types de pratiques et production de lieux.

\section{QUELQUES OUVERTURES D'UNE APPROCHE RENOUVELÉE}

Le déplacement épistémologique de l'approche géographique du tourisme, couramment admis, n'est toutefois pas unanimement reconnu (Dewailly, 2006). Il a pourtant permis la formulation et l'exploration de nombreuses et nouvelles pistes de travail. Et cela ne s'est pas fait tout seul, les rencontres avec d'autres champs scientifiques y contribuant activement. Dans un premier temps, les travaux de sociologie étaient essentiellement encore représentés par ceux de Dumazedier (1963), de Lanfant (1972) voire de Krippendorf (1987). Mais ce sont surtout les travaux de Viard (2000, par exemple) qui alimentèrent, de ce côté, les débats ; travaillant sur le tourisme, il pose aussi, et à par- tir de lui, une série de questions sur les dynamiques sociales d'ensemble. Quant à Kalaora (1993), entre sociologie, histoire et environnement, il croise brillamment mise en tourisme et aménagement forestier. Comme en écho, les travaux des historiens ne furent pas en reste. Joutard (1986), Corbin (1988, 1995), Rauch (1996), Boyer (1996, par exemple) et BerthoLavenir (1999) en sont, parmi quelques autres, les principaux représentants. Le texte de Chadefaud (1987) les rencontre. C'est qu'ils permettent de fructueuses mises en perspective, quitte à singulariser la prise en compte du tourisme par les sciences géographiques. Car les questions sont bien là, et elles concernent tout le monde : le tourisme est-il un phénomène permanent - et dans ce cas, pourquoi ne pas considérer qu'Ulysse ou Montaigne sont déjà des touristes - ou, en tant que phénomène social, est-il lié à un moment historique, en l'occurrence, celui de la première révolution industrielle, de son urbanisation et du développement simultané des moyens de transports ? Enfin, il ne faudrait surtout pas omettre les textes des anthropologues, particulièrement ceux d'Urbain (1993,1994, par exemple). Et encore aura-t-on lu avec grand écho et profit les recherches de Le Menestrel (1999) montrant, à propos des Cadiens de Louisiane, à quel point le tourisme favorisa l'émergence d'une culture propre, non plus repliée sur elle-même, mais mise en tourisme, intégrée à la société globale dans laquelle elle s'inscrit.

Finalement, et parmi plusieurs autres, toute cette effervescence autour du tourisme aura permis de faire émerger quelques ouvertures de recherche.

\section{La critique des mots par les lieux, et réciproquement}

Reprenant un idéal des voyageurs de la fin du XVIII ${ }^{e}$ siècle, la critique des discours sur les lieux par l'observation même de ces lieux ouvre des pistes aussi riches qu'inattendues.

Prenons le cas du travail consacré aux Seychelles, déjà un peu ancien certes, mais assez révélateur de ce qui veut être dit. CazesDuvat (1999) y monte un indice d'attractivité et le calcule à propos d'une cinquantaine de plages de l'archipel. Au final, le meilleur résultat est obtenu par l'Anse Lazio, sur l'île de Praslin. L'auteur conclut logiquement à la nécessaire protection du site vis-à-vis du tourisme. Or, considérant les difficultés même d'accès, on comprend mal, finalement, comment un site relativement peu fréquenté peut, en même temps, être le plus attractif. Et le choix - délibéré ou non - de la photographie de la couverture du livre, montrant une plage vide d'hommes, le confirme encore. Dès lors, et pour comprendre l'apparente contradiction, il convient de reprendre la thèse développée par Gay (2004). Le discours protectionniste, fondé sur l'argument écologique, justifie une hausse des prix, donc une augmentation des recettes touristiques, en même temps qu'un tri social des touristes...

Au-delà de cet exemple ponctuel, c'est toute la conception des relations entre tourisme et lieu qui est en cause et, à travers elle, les attitudes scientifiques qui relèvent autant du procès d'intention que de la norme implicite. À la parabole du "bon voyageur", entendons l'élite touristique qui finira bien par payer sans passer, s'opposerait celle du "mauvais 
touriste", représentant grossier d'un tourisme de masse aussi destructeur que le tourisme culturel ou l'écotourisme pourraient être respectueux des lieux et des autres.

Effectivement, la double lecture croisée des lieux et des discours sur les lieux conduit bien à s'interroger sur ces préceptes touristiques. Ils sont fondés sur les chartes directement issues de l'application du développement durable au tourisme. Car si quelques lieux sont visiblement très fréquentés - certains diront qu'ils le sont trop -, la plupart des autres, qui ne le sont pas tant que cela, parfois le déplorent. Et encore, l'enjeu n'est pas que quantitatif. Les réflexions de l'Unesco en la matière en attestent directement. Dans sa critique de la notion de "capacité de charge touristique", Pedersen (2002) fait ouvertement valoir qu'un touriste malveillant peut faire, à lui seul, plus de "dégâts" que des dizaines d'autres, respectueux des lieux où ils se trouvent et de leurs habitants. D'où la nécessité de changer de modèle d'aménagement et de gestion et l'émergence de la notion de "limite du changement acceptable" (2002, p. 57) : "The challenge is to define how much and what kind of change in 'acceptable" "[...]." Cela implique une vision beaucoup plus souple et dynamique, sans limites en soi, mais avec des seuils qui varient suivant des paramètres eux-mêmes ajustables (2002, p. 58), "for example, the number of guards available for patrolling the site to insure its protection". Tout cela confirme que la responsabilisation des touristes est, à moyen terme, socialement, économiquement et encore humainement, préférable aux interdictions, comme le suggère du reste Knafou (2003).
Une approche par les pratiques

Considérer le tourisme par les touristes, c'est donc privilégier le point de vue des pratiques. Partant de la définition qu'en donne Michel Foucault (2004, p. 22), à savoir ce que "les hommes font et comment ils le font". On y ajoutera le "où" ils le font.

C'est de ce point de vue que l'on peut apprécier le cas de Benidorm (équipe MIT, 2005). Souvent décrit comme l'abominable station des masses, le cas de cette ville est pourtant singulier. D'une part, la fréquentation, près de quatre millions de visiteurs par an, ne se dément pas; de l'autre, elle se diversifie même, les retraités britanniques occupant les lieux l'hiver durant.

Bref, pour qui veut comprendre ce qui se passe, touristiquement, à Benidorm, mieux vaut aborder le lieu à partir des pratiques qui s'y déroulent que d'en lire, ici ou là, les appréciations livrées à l'emporte-pièce. De fait, Benidorm ne donne à voir ni les choix des aménageurs, ni les discours des élites sur le tourisme de masse, mais une série de choix et d'usages sociaux qui font bien partie du monde contemporain. Voilà donc un fait à bien prendre en compte pour qui cherche à comprendre les dynamiques sociales à l'œuvre, avant que de penser les interpréter.

Autrement dit encore, si les données statistiques, prises pour ellesmêmes, n’ont guère de portée, leur croisement avec des observations plus qualitatives s'avère le plus sûr moyen de cerner, au plus près qu'il se peut, de telles situations. L'importance, tout autant que la constance, de la fréquentation de certains lieux n'est peut-être pas seulement liée à "l'instinct grégaire" de touristes lobotomisés par les campagnes publicitaires, mais aussi à des choix combinant toujours subtilement les dimensions sociale et personnelle.

\section{L'historicité des lieux et du tourisme}

Les lieux changent, bougent et se transforment. Et le tourisme y participe, peut-être même au premier chef. Et cela s'observe couramment : c'est, par exemple, le cas du Pouliguen, aujourd'hui station littorale de l'Atlantique breton, qui était, dès avant la Première Guerre mondiale, une station touristique associée à la bourgeoisie urbaine et conquérante des débuts du xxe siècle, puis fut un peu délaissée, comme tant d'autres, après 1945 ; cela dit, peu à peu au cours des années 1990, le lieu a été réinvesti par des populations urbaines, désormais sensibles à ces qualités qu'ont valorisées et promues les activités touristiques précédentes. Dans ce cas précis, une telle dynamique s'accompagne d'un véritable remodelage territorial, notamment soutenu par l'arrivée du TGV en gare du Croisic. Ainsi a émergé, en janvier 2003, une communauté de quinze communes, à cheval sur deux départements, la LoireAtlantique et le Morbihan, ce qui n'est pas si courant. Entre La Baule, Le Croisic et Guérande, on la connaît désormais sous le nom de Cap Atlantique. Ironiquement, les transformations qui ont suivi la catastrophe de l'Erika en 1999 ont finalement participé au renforcement des aménagements touristiques, en particulier le long du littoral et de ses sentiers.

Au-delà de ses propres intérêts, l'approche géographique du tourisme aura donc participé à renouveler la conception de la notion générale de lieu. En évoquant la dynamique de leurs "itinéraires", elle ouvre aussi la perspective plus 
générale des lieux éphémères aussi bien que celle des lieux mobiles, ceux des croisières touristiques par exemple. Les touristes se déplacent, certes, mais ils déplacent aussi et en partie les lieux d'où ils viennent, comme Deprest (1997) l'analysait en utilisant la notion de transfert, en l'occurrence le transfert des centralités urbaines dans le cas des débuts du tourisme. Bref, si le tourisme a eu ses débuts, il aura très probablement une fin et l'on peut, d'ores et déjà et très probablement, s'en figurer quelques contours. C'est que, à Cap Atlantique comme auparavant dans certaines parties de l'Île-deFrance, on assiste clairement à un phénomène que l'on pourrait qualifier de "métropolisation" des territoires. Leurs habitants urbains résident ici et travaillent là et, dans ce schéma, les anciennes stations touristiques sont habitées par des populations régulièrement mobiles. On pourrait même se demander si ce modèle ne vaut pas, au-delà du cas français, comme l'un de ceux de la construction territoriale européenne (Lazzarotti, 2007).

\section{QUELQUES PISTES À EXPLORER}

Ainsi, l'aventure de l'approche géographique du tourisme n'aura sans doute pas tant épuisé le thème que suggéré ceux qui pourraient rester à venir. Chacun y trouvera sans doute les siens, mais, parmi eux, certains peuvent apparaître, à ce jour en tout cas, plus probables que d'autres.

Une problématique existentielle

Quels sont les effets du tourisme, autrement dit des expériences touristiques (Laplante, 1996), sur les touristes eux-mêmes ? Parcourir le monde, c'est éprouver, simultané- ment, l'autre et l'ailleurs (Cériani, Duhamel, Knafou et Stock, 2005). Mais, tout autant, c'est s'éprouver soi-même face aux autres et ailleurs. Et cela n'est jamais aisé. De fait, ces expériences de déplacement peuvent se révéler d'un apprentissage difficile. Du coup, certaines structures - faut-il parler d'aménités ? -, plutôt que de passer pour de véritables sous-produits d'une culture mondiale insipide, pourraient bien valoir comme aides à la mobilité. Quand la nourriture locale est à ce point étrangère, la restauration rapide permet, dans un premier temps du moins, de s'alimenter en attendant l'acquisition d'autres familiarités. Il en va de même des voyages en groupe, facilitant les premières prises de contact avec des lieux lointains.

Le résultat de tous ces efforts est, objectivement, un enrichissement de la "carte d'identité" des touristes par augmentation du nombre de lieux fréquentés. Cette dynamique existentielle est, à sa manière donc, double. D'un côté, elle est celle d'une singularisation des habitants par leurs pratiques touristiques ; de l'autre, elle participe encore à leur mondialisation, soit à l'accès à la composante mondiale de la part géographique de leur identité. Du coup, et de manière plus générale, que ce soit par le tourisme ou une tout autre pratique, il n'y a pas d'identité qui, réciproquement et de manière quasi mécanique, ne mette aussi en cause l'altérité.

\section{Une problématique politique}

La problématique politique est celle de la rencontre et de son symétrique implicite, le contrôle de la mobilité : comment bouger dans le monde, sans le changer?

Plus, très probablement, que n'importe lequel des autres argu- ments, le tourisme joue sur les promesses des rencontres heureuses, ce que ne dément certainement pas le cas de Benidorm. De ce point de vue, il est un formidable ressort de mobilités. Cela dit, toute rencontre est, à chaque fois, susceptible d'effets incontrôlables, politiquement incontrôlables s'entend. En particulier, elle peut changer ceux qui la font. Du coup, et cela vaut pour tout type de rencontre dans tout lieu, ces effets peuvent représenter un danger politique, en particulier et dans un premier temps, du point de vue des tenants de l'ordre des lieux.

On peut ainsi comprendre les tentatives permanentes - entendons, pour ce qui nous concerne, aussi vieilles que le tourisme - afin d'en maîtriser les pratiques, pensant par là en contrôler les effets. Cela ne se fait pas seulement par des dispositifs matériels, comme le contrôle des accès, mais cela passe aussi par un travail idéologique et, aujourd'hui, théoriquement argumenté par le développement durable.

Nous avons déjà montré comment, en son nom, était légitimé l'affirmation d'une norme touristique, fondée en partie sur la culpabilisation des touristes, voire leur infantilisation. La problématique environnementale croise donc le politique et se noue encore dans l'existentiel, en limitant - ou plus exactement en tentant de le faire - l'accès possible à l'autonomie des habitants par les pratiques touristiques. En même temps, en construisant dans la contradiction pratiques touristiques et conservation des lieux, ces discours détournent, par d'apparentes évidences, les touristes d'une critique d'un modèle touristique valorisant le tourisme culturel et son pendant de "nature", l'éco-tourisme. 
Et cela les éloigne encore plus de l'un des véritables enjeux du tourisme - et l'un de ses principaux arguments, s'il n'était le seul : la rencontre avec d'autres. Et, en se fondant dans le "durable" au point de s'y confondre, c'est la norme d'un tourisme un peu aveugle sur lui-même qui tend à s'imposer, dans les discours du moins, comme fragment d'un modèle dominant, idéologiquement dominant, militant et garant d'un ordre du monde (Lazzarotti, 2007).

\section{Une problématique économique}

Le tourisme est-il le pire ennemi du capitalisme, en promouvant ses contre-valeurs, le temps libre contre le travail par exemple, ou son meilleur allié, en promouvant, à la limite subrepticement, quelquesunes de ses alternatives possibles?

Après celui de Las Vegas et de quelques autres, le cas de Dubaï, célèbre pour ces exubérances et, désormais, pour ses difficultés, permet d'alimenter de manière assez claire le débat. D'un côté, Dubai peut être décrit comme un pire des mondes (Davis, 2007), où s'exhibe sans retenue une ségrégation sociale radicale. Mais, de l'autre, le lieu peut aussi faire figure de modèle économique alternatif à celui d'une première révolution industrielle fondé sur l'exploitation des richesses du sol et du sous-sol. De fait, Dubaï localise au mieux dans le monde la mise en application du modèle de l'économie "présentielle", telle que Davezies et Lejoux (2003) en donnent une idée. Il ressort que ce sont les hommes et les femmes qui, par leur présence et leurs échanges sur place, produisent la richesse du lieu. Bref, contre-modèle de la société industrielle, le tourisme s'impose parfois comme fondement même d'un modèle économique dominant. Et cela repose donc bien sur un alliage paradoxal, politiquement conservateur, d'une part, et économiquement libéral, de l'autre.

Le cas de Dubaï montre que le paradoxe n'est peut-être pas exceptionnel, mais qu'il vaut comme les bases mêmes de ce capitalisme contemporain, et rappelle, au passage, l'importance du contrôle politique des mobilités : effectivement, comment bouger dans le monde sans changer le monde, sans changer son ordre politique ?

\section{Une problématique mémorielle}

La Convention du patrimoine mondial, de 1971, après avoir été quelques fois réajustée, rejoint désormais directement le champ des problématiques touristiques (Lazzarotti, 2003 ; Lazzarotti et Violier, 2007). Ses lieux ont partie totalement liée avec le tourisme, comme le soulignait clairement Francesco Bandarin, alors directeur de l'Unesco (Pedersen, 2002, p. 3) : “[... ] Les raisons fondamentales pour lesquelles un bien est inscrit sur la liste du patrimoine mondial sont les mêmes que celles pour lesquelles des millions de touristes les visitent chaque année." Cela donne naissance à des types de lieux singuliers, que l'on pourrait qualifier de "lieux-mondes" : totalement locaux, parce que valorisant un patrimoine par définition unique, totalement mondiaux parce que traversés par des visiteurs du monde, même si, pour être juste, il faut noter que ces visiteurs ne viennent pas du monde entier. Autrement dit, le patrimoine localise ce que le tourisme mondialise.

La notion de patrimoine prend ainsi, et peu à peu, son sens actuel. Car, en même temps qu'elle s'affirme à partir des années 1970, s'estompe symétriquement celle de monument Entre les deux, il n'y a pas qu'une différence de contenu, mais un changement radical dans la manière de produire les mémoires du monde. Les mots des élites savantes qui avaient fait les monuments sont désormais dépassés par les pratiques des touristes qui, présents dans les lieux, les rendent visibles par leur propre regard. Normalement, on peut encore saisir un changement de sens : le patrimoine désigne désormais ce qui, de la nature et de l'histoire, valorise la part non "délocalisable" des lieux, pour cultiver les rouages mêmes de l'économie présentielle : le choix des lieux qui est celui des touristes a désormais son corollaire, leur concurrence...

\section{CONCLUSION : TOURISME, CHANGEMENTS SOCIAL ET SCIENTIFIQUE}

L'apport du tourisme aux sociétés ou, plus précisément, les changements sociaux que le tourisme aura portés, sont considérables. Phénomène marginal, il entre aujourd'hui dans le champ du quotidien, du moins dans celui des sociétés riches d'Occident. C'est en cela que Michel Lussault (2007) peut le qualifier de "genre commun" : comment, dès lors, le tourisme ne conduirait-il pas, au-delà de lui-même ou de ce qu'il en reste, à poser une partie importante des grandes questions du monde contemporain?

Son intérêt pour les sciences sociales n'est certainement pas démenti. Assurément, certaines d'entre elles s'y sont manifestées plus amplement que d'autres, plus discrètes, comme la sociologie, et cela malgré une récente publication affichant clairement son intérêt envers ce "nouvel objet pour les sciences sociales" (Cousin et Réau, 2009)... Peut-être faut-il y 
voir, comme le souligne Poche (1996), ses réserves à l'égard de ce qui implique (aussi) l'espace, "continent noir de la sociologie". Les raisons seraient alors inverses de ce qu'elles furent, positivement, pour la géographie. Le tourisme parle des lieux, il parle des habitants et de leurs mobilités. Du coup, les études sur le tourisme ne risquent pas d'en épuiser le champ, et ce, pour une raison qui tient au tourisme lui-même : il est changements, renouvellements et transformations en cours (Sacareau, 1997). En promouvant les mobilités, le tourisme, pédagogie du monde, épreuve de soi et des autres dans ses lieux et territoires, bouleverse des siècles de sédentarité dominante et participe, pour sa part, à l'émergence des "sociétés à habitants mobiles" dont il procède tout autant.

Cette boucle jamais achevée mais est-elle achevable ? - invite réflexivement à un renversement de la lecture du monde et au changement d'horizon d'une science géographique désormais attentive à l'analyse des mobilités, non plus d'un point de vue unique, mobiliste ou immobiliste, mais dans leurs relations mêmes. D'où l'un des intérêts du concept d'"habiter" et l'une de ses raisons d'être (Lazzarotti, 2006). L'heuristique s'y fait analyse avant que d'oser l'interprétation. Car le langage silencieux de la géographie n'est certainement pas muet, pour qui veut l'entendre. Dès lors, la matière dont il s'agit, celle des lieux et de leurs formes, celle des corps tout aussi bien, dont on peut suivre les mouvements dans le monde et les pratiques dans les lieux, prend toutes ses raisons : comment habiter le monde ou comment être soimême, géographiquement, parmi les autres ? De fait, cette matière, constituée par les pensées et les sentiments des hommes s'écrivant dans le monde et l'écrivant aussi, est en même temps matière première possible de la science géographique. Par son travail encore, elle en est la matière à penser.
(*) Ce travail est la mise en texte d'une intervention faite dans le cadre du séminaire "Épistémologie des études touristiques", tenu au département des études touristiques et urbaines de l'Uqam, le 26 octobre 2009.

\section{RéFÉRENCES BibliographiQues}

Rachid AmIROU et Philippe BACHIMON, Le Tourisme local. Une culture de l'exotisme, coll. Tourismes et sociétés, L'Harmattan, 2000, 238 p.

Yvette BARBAZA, Le Paysage humain de la Costa Brava, éd. Armand Colin, 1966, 718 p.

Nacima BARON-YeLLĖs, Le Tourisme en France. Territoires et stratégies, coll. Prépas-Géographie, éd. Armand Colin, 1999, $220 \mathrm{p}$.

Mohamed BERRIANE, Tourisme national et migrations de loisirs au Maroc (étude géographique), Thèses et mémoires, université Mohammed $\mathrm{V}$ (Rabat), publications de la faculté des lettres et sciences humaines, série $n^{\circ}$ 16, 1992,498 p.

Catherine BeRTHO-LAVENIR, La Roue et le stylo. Comment nous sommes devenus touristes, coll. Champ médiologique, éd. Odile Jacob, Paris, 1999, 438 p.

Jean-Pierre BESANCENOT, Climat et tourisme, coll. Géographie, éd. Masson, 1990, 223 p.

Roger BÉTEILle, Le Tourisme vert, coll. Que sais-je ?, n 3191, PUF, 1966, $128 \mathrm{p}$.

Michel Bonneau, Le Fait touristique dans la France de l'Ouest: contribution à une recherche sur le tourisme rural, université de Rennes, 3 vol., 1978, 1490 p.

Marc BOYER, L'Invention du tourisme, coll. Découvertes, Gallimard, Paris, 1996, 160 p.

Louis BURNET, Villégiature et tourisme sur les côtes de France, thèse dactylographiée, 3 tomes, la Sorbonne, 1957, 502, 792 et 1309 p.

Maurice CAPOT-REY, Géographie de la circulation sur les continents, coll. Géographie humaine, n²0, NRF Gallimard, 1947, 296 p.

Micheline Cassou-Mounat, La Vie humaine sur le littoral des Landes de Gascogne, 2 vol., Atelier de reproduction des thèses, Lille, 1977, 590 et $480 \mathrm{p}$.

Georges CAZES, Les Nouvelles Colonies de vacances ?, 2 tomes, L'Harmattan, 1989 et 1992, 336 et 208 p.

Georges CAZES, Fondements pour une géographie du tourisme et des loisirs, coll. Amphi-géographie, Bréal, Paris, 1992, 189 p. 
Georges CAZEs et Françoise Potier, Le Tourisme et la ville. Expériences européennes, coll. Tourismes et sociétés,

L'Harmattan, 1998, 198 p.

Virginie CAZEs-Duvat, Les Littoraux des Îles Seychelles, L'Harmattan, 364 p.

\section{Giorgia CÉrIANI, Philippe Duhamel, Rémy Knafou et Mathis}

STOCK, "Le tourisme et la rencontre de l'autre. Voyage au pays des idées reçues", L'Autre, cliniques, cultures et sociétés, vol. 6 , $n^{\circ}$ 61, 2005, pp. 71-82.

George Снавот, “L'évasion urbaine”, La Vie urbaine, Dunod, avril-juin 1957, pp. I08-I 6.

Michel Chadefaud, Aux origines du tourisme dans les pays de l'Adour. Du mythe à l'espace : un essai de géographie historique, Cahiers de l'université de Pau, 1987, 1010 p.

Daniel CLARY, La Façade littorale de Paris, éd. Ophrys, 1977. 383 p.

Daniel CLARY, Le Tourisme dans l'espace français, coll. Le Géographe, Masson, 1993, 358 p

Alain CORBIN, Le Territoire du vide. L'Occident et le désir du rivage, 1750-1850, coll. historique, Aubier, 1988, 412 p.

Alain CoRBIN (dir.), L'Avènement des loisirs, 1850-1960, Aubier, 1995, $472 \mathrm{p}$

Saskia Cousin et Bertrand RÉAu, Sociologie du tourisme, coll. Repères, La Découverte, 2009, 126 p.

Françoise CRIBIER, La Grande Migration d'été des citadins en France, Mémoires et documents, éd. du CNRS, Paris, numéro hors série, 1969, 403 p.

Étienne DaLmasso, "Nice, station touristique et grande ville régionale", L'Information géographique, nº 2, 1963, pp. 69-79.

Laurent DAVEZIES et Patricia Lejoux, "Un train peut en cacher un autre. Derrière l'économie productive, l'économie présentielle", dans Concentration et ségrégation, dynamiques et inscriptions territoriales, XXXIXe colloque de l'ASRDLF, Lyon, Ier, 2 et 3 septembre 2003, 17 p.

Mike Davis, Le Stade Dubaï du capitalisme, coll. Penser/croiser, Les Prairies ordinaires, 2007, 88 p.

Bernard DebarbieuX, Tourisme et montagne, coll. Géopoche, éd. Économica, 1995, 100 p.

Florence DEPREST, Enquête sur le tourisme de masse. L'écologie face au territoire, coll. Mappemonde, Belin, 1997, 205 p.

Jean-Michel Dewalluy, Tourisme et loisirs dans le Nord - Pas-deCalais. Approche géographique de la récréation dans une région urbaine et industrielle de l'Europe du Nord-Ouest, 2 tomes,

Société de géographie de Lille, 1985, I |6 | p.

Jean-Michel DeWAlLly, Tourisme et aménagement en Europe du Nord, coll. Géographie, Masson, 1990, 148 p.

Jean-Michel DeWAILly, Tourisme et géographie, entre pérégrinité et chaos? coll. Tourismes et sociétés, L'Harmattan, 2006, 222 p. Jean-Michel DeWAIlly et Émile Flament, Géographie du tourisme et des loisirs, coll. D.I.E.M., Sedes, 1993, 287 p. Jean-Michel Dewalluy et Émile Flament, Géographie du tourisme et des loisirs, coll. Campus Géographie, Sedes, 2000, $192 \mathrm{p}$.

Philippe Duhamel, Les Résidents étrangers européens à Majorque (Baléares). Pour une analyse de la transformation des lieux touristiques, thèse de géographie, université de Paris-VII, 1997, 717 p.

Philippe DuHAmel et Isabelle SACAREAU, Le Tourisme dans le monde, coll. Prépas-Géographie, éd. Armand Colin, 1998,

$222 \mathrm{p}$.

Joffre DuMAZEDIER, Vers une civilisation des loisirs?, coll. Esprit, 1963, 318 p.

ÉQUIPE MIT, Tourismes I, lieux communs, coll. Mappemonde, éd. Belin, 2002, 320 p.

ÉQUIPE MIT, Tourismes 2, moments de lieux, coll. Mappemonde, éd. Belin, 2005, $350 \mathrm{p}$.

Jean-Christophe GAY, "Tourisme, politique et environnement aux Seychelles", Revue Tiers Monde, t. XLV, $n^{\circ}$ I78, avril-juin 2004, pp. 319-339.

Jean GINIER, Les Touristes étrangers en France pendant l'été, éd. M.-Th. Genin, 1969, 64I p.

Jacky HeRBIN, Le Tourisme au Tyrol autrichien, 2 tomes, éd. des Cahiers de l'Alpe, 1980, 718 p.

Mimoun Hillalı, Le Tourisme international vu du sud. Essai sur la problématique du tourisme dans les pays en développement,

Presses de l'université du Québec, 2003, 228 p.

Mimoun HILlali, La Politique du tourisme au Maroc, diagnostic, bilan et critique, coll. Histoires et perpectives méditerranéennes, L'Harmattan, 2007, 294 p.

Philippe JoutARD, L'Invention du Mont-Blanc, coll. Archives, Gallimard/Julliard, 1986, 224 p.

Bernard KALAORA, Le Musée vert. Radiographie du loisir en forêt, coll. Environnement, L'Harmattan, 1993, 304 p.

Rémy KNAFOU, Les Stations intégrées de sports d'hiver des Alpes françaises, Masson, 1978, 319 p.

Rémy KNAFOu, "Vers un tourisme responsabilisé", dans Mathis STOCK (dir.), Le Tourisme, acteurs, lieux et enjeux, coll. Belin Sup Géographie, Belin, 2003, 304 p., pp. 209-253.

Rémy KNAFOU et Mathis STOCK, "Tourisme", dans Jacques LÉVY et Michel LusSAULT, Dictionnaire de la géographie et de l'espace des sociétés, Belin, 2003, I034 p., pp. 931-934.

Rémy KNAfOU, Mireille Bruston, Florence Deprest, Philippe DuHAmel, Jean-Christophe GaY et Isabelle Sacareau, "Une approche géographique du tourisme", Espace géographique, $\mathrm{n}^{\circ}$ 3, 1997, pp. 193-204.

Jost KRIPPENDORF, Les Vacances et après? Pour une nouvelle compréhension des loisirs et des voyages, coll. Logiques sociales, L'Harmattan, 1987, 238 p. 
Marie-Françoise LANFANT, Les Théories du loisir. Sociologie du loisir et idéologie, coll. Le Sociologue, PUF, 1972, 256 p.

Marc LAPLANTE, L'EXpérience touristique contemporaine. Fondements sociaux et culturels, Presses de l'université du Québec, 1996, 168 p.

Olivier LAZZAROTTI, Les Loisirs à la conquête des espaces périurbains, coll. Tourismes et sociétés, L'Harmattan, 1995, $319 \mathrm{p}$.

Olivier LAZZAROTTI, "French tourism geographies: a review", Tourism Geographies, Routledge, vol. 4, n², may 2002, pp. I35147.

Olivier LAZZAROTTI, "Tourisme et patrimoine : ad augusta per angusta”, Annales de géographie, n 629, janvier-février 2003 , pp. 91 - II0.

Olivier LAZZAROTtI, Habiter, la condition géographique, coll. Mappemonde, Belin, 2006, 288 p.

Olivier LazZAROtTI, "Aux horizons du monde : durable ou habitable ?", Revue des Deux Mondes, octobre-novembre 2007. pp. I I 8- 127.

Olivier LAZZAROTTI, “L'habiter touristique et la ville", dans Philippe Duhamel et Rémy KNAFOu, Mondes urbains du tourisme, coll. Mappemonde, Belin, 2007, 366 p., pp. 323-322.

Olivier LAZZAROTtI et Philippe VIOlier, Tourisme et patrimoine, un moment du monde, Actes du colloque de la commission de géographie du tourisme du Comité national de géographie, tenu à Saumur en mai 2004, Presses de l'université d'Angers, 2007, 248 p.

Maurice Le LANNOU, Itinéraires de Bretagne. Guide géographique et touristique, éd. Baillière et fils, 1938, 284 p. Sara Le Menestrel, La Voie des Cadiens, tourisme et identité en Louisiane, coll. Histoire et société, cultures américaines, Belin, 1999, $432 \mathrm{p}$.

Jean-Pierre LozATO-GıOTART, Géographie du tourisme, coll. Géographie, Masson, 4 e éd., 1993, 309 p. (Ire édition, 1985)

Michel LusSAULT, "Le tourisme, un genre commun", dans Philippe Duhamel et Rémy KNafou, Mondes urbains du tourisme, coll. Mappemonde, éd. Belin, 2007, 366 p.,

pp. 333-349.

Pierre MerLIN, Tourisme et aménagement touristique. Des objectifs inconciliables ?, La Documentation française, 200 I, $216 \mathrm{p}$.

Jean-Luc Michaud, Le Tourisme face à l'environnement, coll. Le Géographe, PUF, 1983, 234 p.

Jean-Marie Mıossec, Le Tourisme en Tunisie. Un pays en développement dans l'espace touristique international, université de Tours, 1996, 1333 p.
Joël MIRLoup, Les Fonctions touristiques et de loisirs en Loire moyenne. Contribution à l'étude de l'aire de loisirs des Parisiens, ADCESL, 1981, 846 p.

Douglas PEARCE, Géographie du tourisme, coll. Fac géographie, Nathan, 1993, 352 p.

Arthur Pedersen, Managing tourism at World Heritage Sites, Unesco, 2002, 104 p.

Bernard PocHE, L'Espace fragmenté. Éléments pour une analyse sociologique de la territorialité, coll. Villes et entreprises, L'Harmattan, 1996, 276 p.

Pierre Py, Le Tourisme, un phénomène économique, La Documentation française, $n^{\circ}$ 526I, 2007, I 84 p.

André RAUCH, Vacances en France de 1830 à nos jours, coll. La Vie quotidienne, I'histoire en marche, Hachette, 1996, $280 \mathrm{p}$.

Isabelle SACAREAU, Porteurs de l'Himalaya. Le trekking au Népal, coll. Mappemonde, Belin, 1997, 27I p.

Mathis Sтоск, Mobilités géographiques et pratiques des lieux. Étude théorico-empirique à travers deux lieux touristiques anciennement constitués : Brighton \& Hove (Royaume-Uni) et Garmisch-Partenkirchen (Allemagne), thèse de l'université de Paris VII-Denis-Diderot, 200 I, 664 p.

Mathis Sтоск (dir.), Le Tourisme, acteurs, lieux et enjeux, coll. Belin Sup Géographie, Belin, 2003, 304 p.

Jean-Didier URBAIN, L'Idiot du voyage. Histoires de touristes, Payot, 1993, $270 \mathrm{p}$.

Jean-Didier URBAIN, Sur la plage. Mœurs et coutumes balnéaires, coll. Essais, Payot, 1994, 376 p.

Jean VIARD, Court traité sur les vacances, les voyages et I'hospitalité des lieux, coll. Intervention, éditions de l'Aube, 2000, 170 p.

Philippe VIOLER, Tourisme et développement local, Coll. Belin Sup. Tourisme, éd. Belin, 2008, 192 p.

Gabriel WACKERMANN, Les Loisirs dans l'espace rhénan, de la région zurichoise à la frontière germano-néerlandaise. Une étude géographique d'un espace multinational, Atelier de reproduction des thèses de l'université de Lille III, 1973, 673 p. 